10

\title{
Моделирование оптоакустических сигналов в системе с упругим рассеянием и поглощением света
}

\author{
(C) М.В. Ананьева ${ }^{1}$, А.А. Звеков ${ }^{2}$, А.В. Каленский ${ }^{1}$ \\ ${ }^{1}$ Кемеровский государственный университет, Кемерово, Россия \\ ${ }^{2}$ Федеральный исследовательский центр угля и углехимии СО РАН, Кемерово, Россия \\ E-mail: kriger@kemsu.ru
}

Поступило в Редакцию 9 октября 2018г.

В окончательной редакции 10 января 2019г.

Принято к публикации 11 января 2019г.

\begin{abstract}
Предложена методика и выполнено моделирование оптоакустических сигналов, инициируемых лазерным импульсом, в поглощающей среде с упругим рассеянием излучения. Показано, что использование метода сферических гармоник позволяет получить аналитическое выражение для оптоакустического сигнала. Отклонение профиля поглощения от бугеровского вида вблизи поверхности образца приводит к сильному влиянию параметров среды на положение максимума сигнала. Рост альбедо однократного рассеяния приводит к уменьшению эффективной константы роста сигнала и его амплитуды.
\end{abstract}

DOI: 10.21883/PJTF.2019.07.47538.17554

Оптоакустические методы исследования интенсивно развиваются и широко применяются в дефектоскопии [1] и томографии [2]. В [3] изложены основы оптоакустической спектроскопии, позволяющие связать возникающий в среде акустический отклик с показателем поглощения. Данный метод применяется при исследовании объектов с низкими коэффициентами пропускания, недоступных для просвечивающих методик [3], а также сред с сильным диффузным рассеянием света при слабом поглощении [4]. В [5] оптоакустическим методом определялся эффективный показатель поглощения композитов на основе прозрачной матрицы и наночастиц металлов. В [6] предложен способ оценки показателей поглощения и рассеяния света суспензиями диоксида титана, содержащими краситель метиленовый синий, на основе метода градуировочных графиков для амплитуды сигнала и эффективной константы роста сигнала на начальном участке. Таким образом, перспективность применения оптоакустической спектроскопии к системам, совмещающим упругое рассеяние и поглощение света, подтверждена в ряде работ, при этом теоретическая база метода пока разработана недостаточно. Цель настоящей работы - сформулировать модель оптоакустического отклика в среде, одновременно поглощающей и рассеивающей излучение, и определить, как влияют оптические параметры системы на вид оптоакустического сигнала.

Будем полагать, что среда характеризуется постоянными по объему и не зависящими от интенсивности излучения линейными показателями ослабления $\mu$, рассеяния $\mu_{s c}$ и индикатрисой рассеяния $\chi\left(\xi, \xi^{\prime}\right)$ монохроматического излучения в виде Хеньи-Гринстейна [7] с фактором анизотропии $g$. Формулировку и апробацию модели оптоакустического отклика в среде, одновременно поглощающей и рассеивающей излучение, проведем в одномерном приближении, как в работах $[8,9]$. Для опи- сания распространения монохроматического излучения в светорассеивающей среде при пренебрежении уширением пучка, падающего перпендикулярно на верхнюю поверхность образца, использовалось стационарное одномерное уравнение переноса излучения в виде [8-10]:

$$
\xi \frac{d I(x, \xi)}{d x}=-I(x, \xi)+\frac{\Lambda}{2} \int_{-1}^{1} I\left(x, \xi^{\prime}\right) \chi\left(\xi, \xi^{\prime}\right) d \xi^{\prime}
$$

где $x=\mu z$ - безразмерная координата; $\xi=\cos \theta-$ косинус сферического угла между нормалью к передней границе и направлением визирования; $I(x, \xi)-$ освещенность в точке $x$ в направлении $\xi ; \Lambda=\mu_{s c} / \mu-$ альбедо однократного взаимодействия света с рассеивающей средой. Для решения уравнения (1) использовался метод сферических гармоник: поиск освещенности в виде суперпозиции сферических гармоник [9-12], которые могут быть заменены полиномами Лежандра $\left(P_{l}\right)$. Система уравнений для коэффициентов разложения $C_{m}(x)$ диффузной составляющей освещенности $I_{s}$ по полиномам Лежандра имеет вид [9]:

$$
\begin{aligned}
& \frac{1}{2 m+1}\left[(m+1) \frac{d C_{m+1}}{d \tau}+m \frac{d C_{m-1}}{d \tau}\right] \\
& +\left(1-\frac{\Lambda \chi_{m}}{2}\right) C_{m}=\left(1-R_{f}\right) \frac{\Lambda \chi_{m}}{2} \exp (-x),
\end{aligned}
$$

где $\chi_{m}$ - коэффициенты разложения индикатрисы рассеяния по полиномам Лежандра, $R_{f}-$ коэффициент отражения света при нормальном падении. Для системы (2) использовались френелевские граничные условия $[10,13]$, записанные в матричном виде $[9,10]$. 

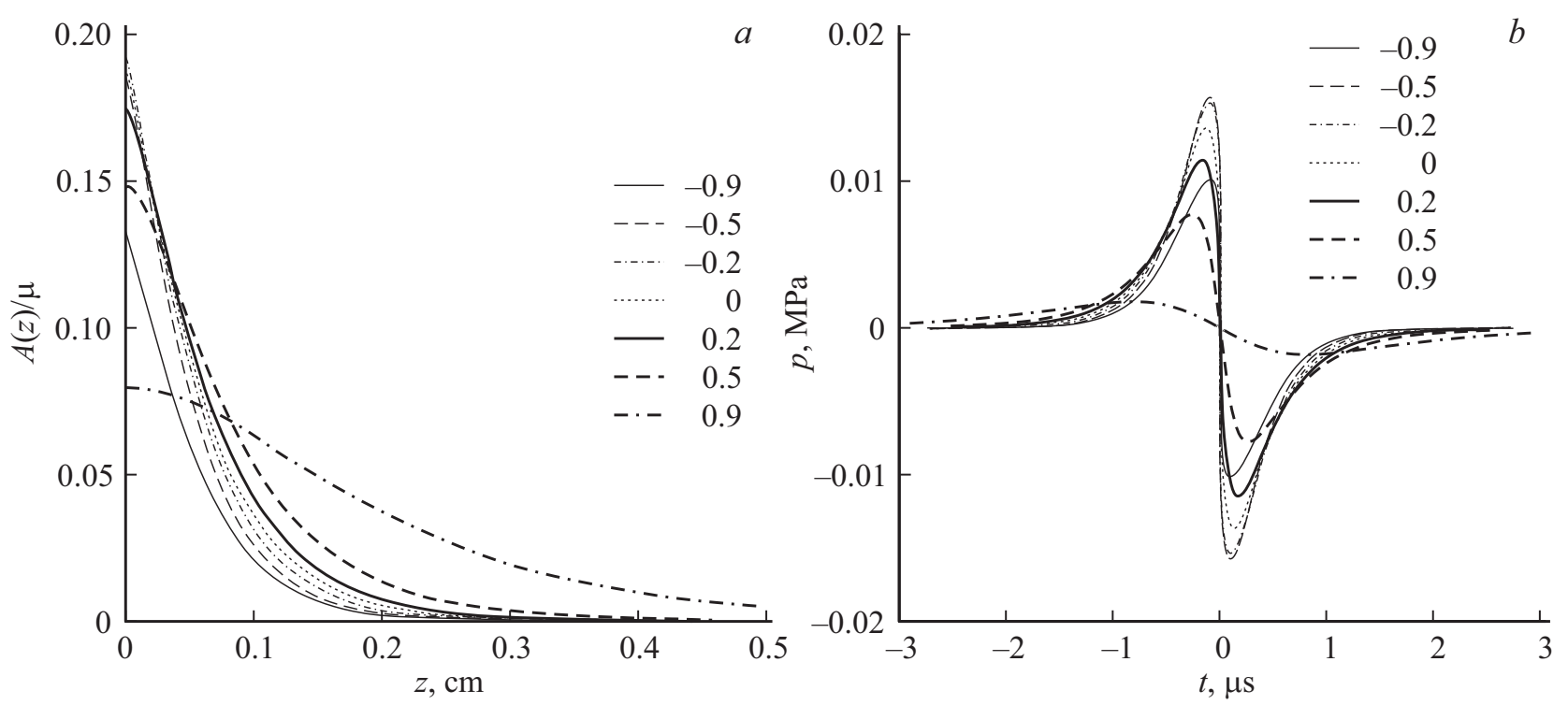

Рис. 1. Рассчитанные распределения поглощенной плотности мощности $(a)$ и зависимости оптоакустических сигналов от времени (b) при $\Lambda=0.95, \mu=50 \mathrm{~cm}^{-1}$ и различных значениях $g$, указанных на рисунке.

Решение системы (2) имеет вид [10]:

$$
C_{m}(x)=\sum_{l} a_{m l} \tilde{C}_{l} \exp \left(-\gamma_{l} x\right)+G_{m} \exp (-x)
$$

где суммирование ведется только по собственным векторам $a_{m l}$ с положительными собственными значениями $\gamma_{l}[14]$. Коэффициенты $G_{m}$ равны

$$
G_{m}=-\left(1-R_{f}\right) \Lambda \sum_{m=0}^{N}\left[\delta_{p m}+A_{p m}\right]^{-1} B_{m},
$$

где

$$
\begin{aligned}
A_{p m} & =-\left[\frac{p+1}{2 p+1} \delta_{p, p^{\prime}+1}+\frac{p}{2 p+1} \delta_{p, p^{\prime}-1}\right]^{-1} \\
& \times\left[\left(1-\frac{\Lambda \chi_{m}}{2}\right) \delta_{p^{\prime} m}\right], \\
B_{m}= & {\left[\frac{m+1}{2 m+1} \delta_{m, m^{\prime}+1}+\frac{m}{2 m+1} \delta_{m, m^{\prime}-1}\right]^{-1}\left[\frac{\chi_{m^{\prime}}}{2}\right] . }
\end{aligned}
$$

Коэффициенты $\tilde{C}_{l}$ определяются через матрицу граничных условий $R_{l m}[10]$ :

$$
\tilde{C}=-\left[(R a)^{T}(R a)\right]^{-1}(R a)^{T}(R G),
$$

где в матрице $a$ оставлены только собственные векторы с положительными собственными значениями. Для распределения поглощаемой плотности мощности по глубине образца $A(z)$, нормированной на падающую величину, получаем выражение

$$
\begin{aligned}
A(z) & =\mu(1-\Lambda)\left(\sum_{l} a_{0 l} \tilde{C}_{l} \exp \left(-\mu \gamma_{l} z\right)\right. \\
& \left.+\left[\left(1-R_{f}\right)+G_{0}\right] \exp (-\mu z)\right),
\end{aligned}
$$

имеющее вид суперпозиции убывающих экспоненциальных функций.

Рассмотрим расчет оптоакустического сигнала в одномерном приближении, пренебрегая процессами диссипации энергии акустической волны [3], когда применимо одномерное волновое уравнение

$$
\frac{\partial^{2} p}{\partial t^{2}}=c_{s}^{2} \frac{\partial^{2} p}{\partial z^{2}}+\frac{\partial S(z, t)}{\partial t}
$$

где $S(z, t)$ - функция источников, соответствующая скорости увеличения давления в среде при отсутствии ее механической релаксации, $c_{s}-$ скорость звука. На значительном отдалении от области поглощения решение уравнения (7) можно записать в виде [3]:

$$
p(t)=\frac{1}{2 c_{s}} \int_{0}^{\infty}\left\{S\left(t+\frac{z}{c_{s}}, z\right)-S\left(t-\frac{z}{c_{s}}, z\right)\right\} d z .
$$

В случае применимости закона Бугера и гауссовой формы импульса функция источников и кинетика изменения давления описываются выражениями [3]:

$$
S=\frac{d c_{s}^{2} \beta \mu_{a b s} H}{\sqrt{\pi} C_{p} \tau_{L}} \exp \left[-\mu_{a b s} z-\frac{t^{2}}{\tau_{L}^{2}}\right],
$$



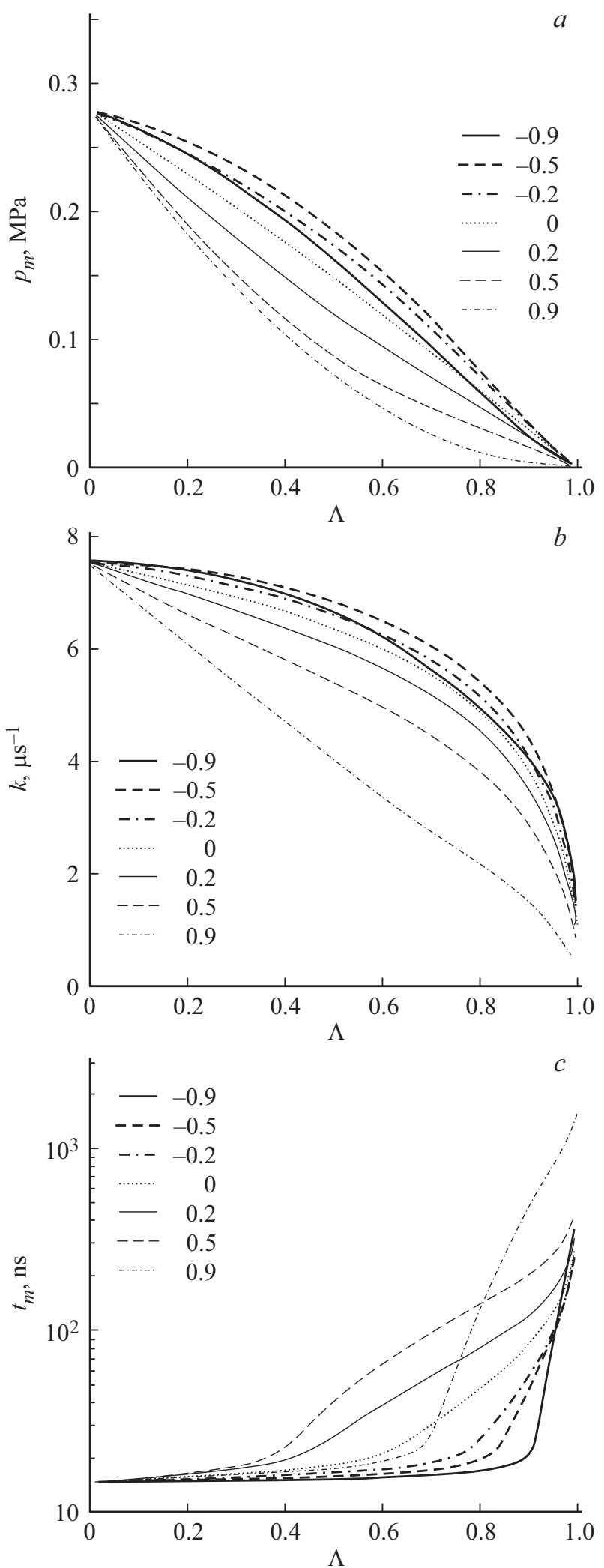

Рис. 2. Рассчитанные зависимости максимального давления в оптоакустической волне $(a)$, эффективной константы роста сигнала на начальном участке $(b)$ и промежутка времени между максимумом оптоакустического сигнала и моментом пересечения зависимостью $p(t)$ оси времени $(c)$ от альбедо однократного рассеяния. Значения фактора анизотропии указаны на рисунке.

$$
\begin{aligned}
& p_{1}\left(t, \mu_{a b s}\right)=\frac{d c_{s}^{2} \beta \mu_{a b s} H}{4 C_{p}} \exp \left[\frac{\left(\mu_{a b s} \tau_{L} c_{s}\right)^{2}}{4}\right] \\
& \times\left\{\exp \left(\mu_{a b s} c_{s} t\right) \operatorname{erfc}\left[\frac{t}{\tau_{L}}+\frac{\mu_{a b s} \tau_{L} c_{s}}{2}\right]\right. \\
& \left.-\exp \left(-\mu_{a b s} c_{s} t\right) \operatorname{erfc}\left[-\frac{t}{\tau_{L}}+\frac{\mu_{a b s} \tau_{L} c_{s}}{2}\right]\right\},
\end{aligned}
$$

где $d-$ плотность образца, $\beta$ - коэффициент термического расширения, $\mu_{a b s}-$ показатель поглощения, $H$ - плотность энергии импульса, $C_{p}-$ объемная теплоемкость, $\tau_{L}$ - половина длительности импульса по уровню $1 / e$ амплитуды. При использовании (10) сигнал в случае свободной границы пересекает ось абсцисс в момент времени $t=0$.

В случае светорассеивающей системы распределение поглощаемой мощности может быть описано суммой слагаемых бугеровского типа (6), и для давления имеем

$$
p(t)=\sum_{l=1}^{0.5 N+1} F_{l} p_{1}\left(t, \mu \gamma_{l}\right)
$$

где при $l \leqslant 0.5 N$ коэффициенты равны $F_{l}=(1-\Lambda) a_{0 l} \tilde{C}_{l}$ с соответствующими собственными числами $\gamma_{l}$, а при $l=0.5 N+1 \quad$ коэффициент $\gamma_{l}=1 \quad$ и величина $F_{l}=(1-\Lambda)\left[\left(1-R_{f}\right)+G_{0}\right]$. Таким образом, использование метода сферических гармоник для решения уравнения переноса излучения позволяет непосредственно рассчитать кинетику оптоакустического сигнала. При расчетах использовались теплофизические параметры воды [15] при $H=0.1 \mathrm{~J} / \mathrm{cm}^{2}$ и $\tau_{L}=8.4 \mathrm{~ns}$.

На рис. 1 показаны рассчитанные распределения $A(z) / \mu(a)$ и соответствующие зависимости оптоакустических сигналов от времени $(b)$ при значении $\Lambda=0.95$, $\mu=50 \mathrm{~cm}^{-1}$ и $g$ от -0.9 до 0.9 . Вдали от границы образца распределение $A(z) / \mu$ близко к экспоненциальному, а вблизи наблюдается значительное отклонение от экспоненциальной функции в меньшую сторону, что типично для диффузно рассеивающих свет систем $[8,10]$. При росте фактора анизотропии происходит увеличение глубины образца, на которую распространяется излучение, и области видимого отклонения от бугеровского профиля поглощения. В результате значение $A$ на поверхности изменяется немонотонно, а коэффициент отражения уменьшается от 62.2 до 9.6\% по мере роста фактора анизотропии. Увеличение $g$ от -0.9 до 0.9 (при $\Lambda=0.95$ и $\mu=50 \mathrm{~cm}^{-1}$ ) приводит к росту времени от максимума сигнала до пересечения оси времени $t_{m}$ от 97.0 до $818.0 \mathrm{~ns}$ и уменьшению эффективной константы роста $k$, определяемой аппроксимацией зависимости $p(t)$ на участке от 5 до 50\% амплитуды экспоненциальной функцией [3-5], сигнала от $3.233 \mu \mathrm{s}^{-1}$ до 2.525 (при $g=0.2$ ) и $0.965 \mu \mathrm{s}^{-1}$ (при $\left.g=0.9\right)$. Амплитуда сигнала зависит от фактора анизотропии немонотонно (рис. $1, b$ ). 
Перейдем к рассмотрению зависимостей характерных параметров оптоакустического сигнала от альбедо однократного рассеяния и фактора анизотропии (рис. 2). Увеличение альбедо приводит к падению амплитуды сигнала (рис. 2,a), эффективной константы его роста (рис. 2, b) и увеличению времени между максимумом и нулевой отметкой на шкале времени $t_{m}$ (рис. 2,c). Уменьшение амплитуды согласуется с увеличением коэффициента отражения $\rho$ благодаря повышению доли диффузной составляющей освещенности. В области малых $\Lambda$ рост фактора анизотропии вызывает монотонное уменьшение эффективной константы роста сигнала, а в области высоких зависимость перестает быть монотонной. Время до максимума $t_{m}$ в основном увеличивается при росте $g$.

Рост альбедо однократного рассеяния приводит к увеличению области поглощения излучения с одновременным ростом коэффициента диффузного отражения. Это естественно приводит к уменьшению эффективной константы роста сигнала и амплитуды максимума. Направление большей части излучения вперед при положительных $g$ приводит также к увеличению области поглощения энергии. Поскольку интеграл от распределения поглощенной плотности мощности, деленной на плотность мощности импульса, равен $1-\rho$, влияние на амплитуду сигнала оказывают оба эффекта: увеличение коэффициента отражения и уменьшение константы роста сигнала при увеличении альбедо. Тенденция к немонотонному изменению амплитуды сигнала и эффективной константы роста при увеличении фактора анизотропии хорошо прослеживается на рис. 2, $a$ и $b$. Этот эффект, а также область значений альбедо и фактора анизотропии вблизи 0.9 будут подробно рассмотрены в следующих публикациях.

Наличие зависимостей трех параметров сигнала $\left(p_{m}\right.$, $t_{m}$ и $k$ ) от трех параметров объекта $(\mu, \Lambda$ и $g)$ свидетельствует о перспективности использования оптоакустической спектроскопии для решения обратных задач (например, одновременного определения показателей поглощения и рассеяния образца). В то же время зависимости параметров сигнала от фактора анизотропии являются немонотонными (рис. 2), что может приводить к ослаблению корректности постановки обратной задачи. Повышение корректности может быть достигнуто за счет измерения коэффициента отражения, который не чувствителен к величине показателя ослабления. Другой возможностью является использование нескольких значений длины волны излучения при известном спектре поглощающего свет компонента.

Таким образом, показана принципиальная возможность учета многократного рассеяния света в поглощающей среде в рамках метода сферических гармоник при прогнозировании параметров оптоакустического сигнала. Трехмерный вариант модели оптоакустических явлений и учет различных моделей индикатрисы рассеяния в обсуждавшихся в работе средах будут рассмотрены в следующих публикациях.
Работа выполнена в рамках проекта фундаментальных научных исследований (ГП 14) по теме (проекту) „V.49.1.5. Изучение механизмов преобразования энергии электронно-пучкового и лазерного излучения в высокоэнергетических материалах и углях для создания материалов для компонентов и устройств специального назначения“, регистрационный номер АААА-А17117041910150-2 (руководитель Б.П. Адуев) и при поддержке Министерства образования и науки РФ (задание № 3.5363.2017/8.9).

\section{Список литературы}

[1] Симонова В.А., Саватеева Е.В., Карабутов А.А., Карабутов А.А. (мл.), Каптильный А.Г., Ксенобонтов Д.М., Подымова Н.Б. // Вестн. РФФИ. 2014. № 3(83). С. 10-20.

[2] Xia J., Yao J., Wang L.V. // Prog. Electromagn. Res. 2014. V. 147. P. 1-22.

[3] Гусев В.Э., Карабутов А.А. Лазерная оптоакустика. М.: Наука, 1991. 304 с.

[4] Карабутов А.А., Пеливанов И.М., Подымова Н.Б., Скипетров С.E. // Квантовая электроника. 1999. Т. 29. № 3. C. 215-220.

[5] Адуев Б.П., Нурмухаметов Д.Р., Белокуров Г.М., Нелюбина Н.В., Тупицын А.В. // Оптика и спектроскопия. 2018. T. 124. B. 3. C. $404-409$.

[6] Звеков А.А., Нурмухаметов Д.Р., Коржм М.Г., Каленский А.В., Адуев Б.П. // ЖПС. 2017. Т. 84. № 3. С. 391-398.

[7] Henyey L.G., Greenstein J.L. // Astrophys. J. 1941. V. 93. P. 70-83.

[8] Исимару А. Распространение и рассеяние волн в случайно-неоднородных средах. М.: Мир, 1981. Т. 1. C. $176-178$.

[9] Адуев Б.П., Нурмухаметов Д.Р., Белокуров Г.М., Звеков А.А., Каленский А.В., Никитин А.П., Лисков И.Ю. // ЖТФ. 2014. Т. 84. В. 9. С. 126-131.

[10] Каленский А.В., Звеков А.А., Никитин А.П., Газенаур Н.В. // Изв. вузов. Физика. 2016. Т. 59. № 2. С. 87-94.

[11] Edjlali E., Bérubé-Lauziére Y. // J. Quant. Spectrosc. Rad. Transfer. 2016. V. 182. P. 112-118.

[12] Garrett C.K., Haucka C.D. // Comp. Math. Appl. 2016. V. 72. P. $264-270$

[13] Garcia R.D.M. // J. Quant. Spectrosc. Rad. Transfer. 2013. V. 115. P. $28-45$.

[14] Panasyuk G.Y., Schotland J.C., Markel V.A. // J. Phys. A. 2006. V. 39. P. 115-137.

[15] Таблицы физических величин. Справочник / Под ред. И.К. Кикоина. М.: Атомиздат, 1975. 1008 с. 